\title{
Characteristics Evolution of Multiscale Structures in Deep Coal under Liquid Nitrogen Freeze-Thaw Cycles
}

\author{
Yingfeng Sun $\mathbb{D},{ }^{1,2}$ Yixin Zhao $\left(\mathbb{D},{ }^{3,4}\right.$ Yulin Li $\mathbb{D},{ }^{4}$ Nima Noraei Danesh $\mathbb{D}^{4},{ }^{4}$ \\ and Zetian Zhang ${ }^{2}$ \\ ${ }^{1}$ School of Civil and Resource Engineering, University of Science and Technology Beijing, Beijing 100083, China \\ ${ }^{2}$ Key Laboratory of Deep Earth Science and Engineering (Sichuan University), Ministry of Education, Chengdu 610065, China \\ ${ }^{3}$ Beijing Key Laboratory for Precise Mining of Intergrown Energy and Resources, China University of Mining and \\ Technology (Beijing), Beijing 100083, China \\ ${ }^{4}$ School of Energy and Mining Engineering, China University of Mining and Technology (Beijing), Beijing 100083, China
}

Correspondence should be addressed to Zetian Zhang; zhangzetian@scu.edu.cn

Received 17 August 2021; Accepted 22 September 2021; Published 13 October 2021

Academic Editor: Yong liu

Copyright (C) 2021 Yingfeng Sun et al. This is an open access article distributed under the Creative Commons Attribution License, which permits unrestricted use, distribution, and reproduction in any medium, provided the original work is properly cited.

Liquid nitrogen freeze-thaw fracturing has attracted more and more attention in improving the coal reservoir permeability. In order to reveal the impact of liquid nitrogen freeze-thaw on the multiscale structure of deep coal, the multiscale structure evolution law of deep and shallow coal samples from the same seam in the Qinshui coalfield during the liquid nitrogen freezethaw cycling was investigated using NMR $T_{2}$ spectrum, NMRI, and SEM. The connectivity between mesopores and macropores in deep and shallow coal is improved after liquid nitrogen freeze-thaw cycles. The influence of liquid nitrogen freeze-thaw cycles on the structure evolution of deep and shallow coal is the formation and expansion of microscopic fractures. The initial NMR porosity of deep coal is lower than that of shallow coal from the same coalfield and coal seam. The NMR porosity of both the deep and shallow coal samples increases with the increase of the number of freeze-thaw cycles, and the NMR porosity growth rate of the deep sample is lower than that of the shallow sample.

\section{Introduction}

Global reserves of deep coalbed methane (CBM) are approximately $47.6 \times 10^{12} \mathrm{~m}^{3}[1]$, and the development of deep CBM in the United States started early and has been gradually commercialized [2-7]. Although China developed deep CBM production relatively late, it has achieved success in deep CBM development in recent years in the Cainan area of Junggar Basin [8-10], Yanchuannan on the eastern edge of the Ordos Basin, Linfen block, and Zhengzhuang and Shizhuang block of Qinshui Basin [11], indicating great potential for the development of deep CBM production.

The multiscale structure of coal is not only the main flow channel of CBM but also the main factor influencing its development. Qin et al. investigated the pore-fracture characteristics of deep coal with a depth of more than $1000 \mathrm{~m}$ in the Daning-Jixian area using nuclear magnetic resonance
(NMR) and found that the development of macropores and fractures is superior to that of micropores [12]. Gao studied the influence of temperature and stress on the characteristics evolution of pore-fracture structures in deep coal reservoirs in the Linxing block by NMR and found that the deformation of fractures and mesopores is more significant than that of micropores under the same stress and the negative effect of temperature on fractures and mesopores is greater than that of micropores [13]. Xu et al. analyzed the pore structure of medium-high rank coal samples from depths of 100$1800 \mathrm{~m}$ in Yichuan of Shaanxi Province and Shizhuang of Shanxi Province by mercury intrusion, low-temperature liquid nitrogen adsorption, and NMR. Their results show an increase in micropores and a decrease in mesopores and macropores with depth [14]. Lu et al. analyzed the porefracture structure of coal samples from different depths of 14 coal mines in the south of Qinshui Basin and found that 
fracture density is not affected by depth and is distributed between 7 and 15 fractures $/ 5 \mathrm{~cm}$. Compared with shallow coal samples, the average diameter of the pore-throat in deep coal decreases, the volume of micropores increases slightly, and the volume of mesopores and macropores decreases slightly; when the depth exceeds $1000 \mathrm{~m}$, the porosity is generally less than $6 \%[15,16]$. Zheng et al. studied the variation in porosity with depth in Qinshui Basin and found that the porosity of coal in the depth greater than $800 \mathrm{~m}$ is generally less than $6.5 \%$ [17]. Liang et al. studied the pore structure characteristics of samples at different depths in the Huainan coalfield and found that porosity decreased with depth, and the ratio of the macropore specific pore volume to the total specific pore volume is decreasing, while that of micropores is increasing [18]. Zhang found there is a binomial relationship between porosity and effective stress in deep coal reservoirs in the Yushe-Wuxiang block and Shizhuang south block [19].

Due to the low permeability of CBM reservoirs, hydraulic fracturing has become a common method to improve coal seam permeability. However, the solid particles in the fracturing fluid may block the flow channel, and the adsorption of the fracturing fluid on the coal surface and the waterswelling minerals in the coal seam leads to expansion of the coal matrix during the fracturing, thus reducing the permeability of the seam [20]. In addition, hydraulic fracturing consumes large amounts of water, pollutes groundwater, and induces earthquakes [21]. In view of these factors, exploration of new coal seam fracturing technology is urgent. Liquid nitrogen fracturing has attracted increasing attention in recent years [22, 23]. The vaporization and expansion of liquid nitrogen injected into the coal seam, the solidification and expansion of water in the coal seam caused by low temperature, and the thermal stress caused by low temperature will damage and destroy the coal body, and then, the permeability of the coal seam is improved [24]. Qin systematically studied the effect of liquid nitrogen freeze-thaw on the coal pore-fracture structure. He suggested that more freeze-thaw cycles are better than a longer freezing time in improving coal seam permeability [24], and it is the difference in the initial porosity that causes the different increments of permeability under liquid nitrogen freeze-thaw conditions [25]. Sun et al. found that the impact of freeze-thaw on rocks pores and fractures is sandstone $>$ limestone $>$ granite, which is also related to their initial porosities [26]. Using nitrogen adsorption and mercury injection, Qin et al. found that the cumulative seepage pore volume and total pore volume have an exponential relationship with freezing time, and cumulative pore volume has a quadratic relationship with freeze-thaw cycles [27]. Using NMR and SEM, Qin et al. observed that when the freezing time increased from 1 minute to 1 hour, the maximum fracture width increases from $5.56 \mu \mathrm{m}$ to $100 \mu \mathrm{m}$ [28]. Yan analyzed the pore structure of coal samples with different water content before and after liquid nitrogen freeze-thaw cycling using nitrogen adsorption and found that the variation in pore structure increases with increasing water content [29]. Sun et al. found that the pores and fractures become more developed when the freezing temperature decreases under freeze-thaw conditions [30]. Zhai et al. found that freezethaw cycles increase the proportion of mesopores and macropores and reduce the proportion of micropores [31].

Liquid nitrogen freeze-thaw cycling, a nonwater fracturing technology, has many advantages over hydraulic fracturing. In recent years, scholars have conducted a lot of researches on the effect of liquid nitrogen freeze-thaw cycles on the multiscale coal structure, mainly focusing on the effects of the liquid nitrogen freeze-thaw cycle on shallow coal. In view of this, using the NMR $T_{2}$ spectrum, NMRI, and SEM, this paper compares the evolution of multiscale structures in deep and shallow coal after different numbers of liquid nitrogen freeze-thaw cycles to reveal the multiscale structure evolution law of deep and shallow coal in liquid nitrogen freeze-thaw cycling.

\section{Laboratory Experiments: Methodology and Procedures}

2.1. Samples. The two samples used in the experiments were collected from Qinshui coalfield. The HL sample was collected from coal seam no. 15 in the Hengling block, and the SJZ sample was collected from coal seam no. 15 in the Sijiazhuang coal mine, with sampling depths of 1436 and 420 meters, respectively. The sample was prepared by wireelectrode cutting, as shown in Figure 1. The sample used in the liquid nitrogen freeze-thaw experiment is cylindrical, with a diameter of $25 \mathrm{~mm}$ and a length of $50 \mathrm{~mm}$.

\subsection{Multiscale Structure Evolution Experiment of Deep Coal under Liquid Nitrogen Freeze-Thaw Cycles}

2.2.1. Principles of NMR. This paper mainly uses nuclear magnetic resonance imaging (NMRI) and NMR relaxation spectrum. The basic principle of NMRI is to apply the radio frequency pulse, layer selection gradient field, phase encoding gradient field, and frequency encoding gradient field to the test sample; then, the signal source in three dimensions is located through slice selection, phase encoding, and frequency encoding. The signal is then collected and decoded (Fourier transform). Finally, NMRI is achieved according to the relationship between the signal intensity and image gray value (pixels with larger signals are more bright, while those with smaller signals are less bright) [32, 33]. The NMRI flowchart is shown in Figure 2 [34].

The quantitative relationship between transverse relaxation time $T_{2}$ and pore radius $r$ is mainly used to analyze the multiscale structure of coal in the NMR relaxation spectrum analysis $[35,36]$,

$$
r=\rho F_{\mathrm{s}} T_{2}
$$

where $r$ is the pore radius, $\rho$ is the surface transverse relaxation rate $(\mathrm{m} / \mathrm{s})$, and $F_{\mathrm{s}}$ is the shape factor; $F_{\mathrm{s}}=1,2,3$ for the pore shape of the slit, columnar, and spherical, respectively [37].

As shown in Equation (1), the larger the multiscale structure is, the longer the corresponding transverse relaxation time $T_{2}$ is. 


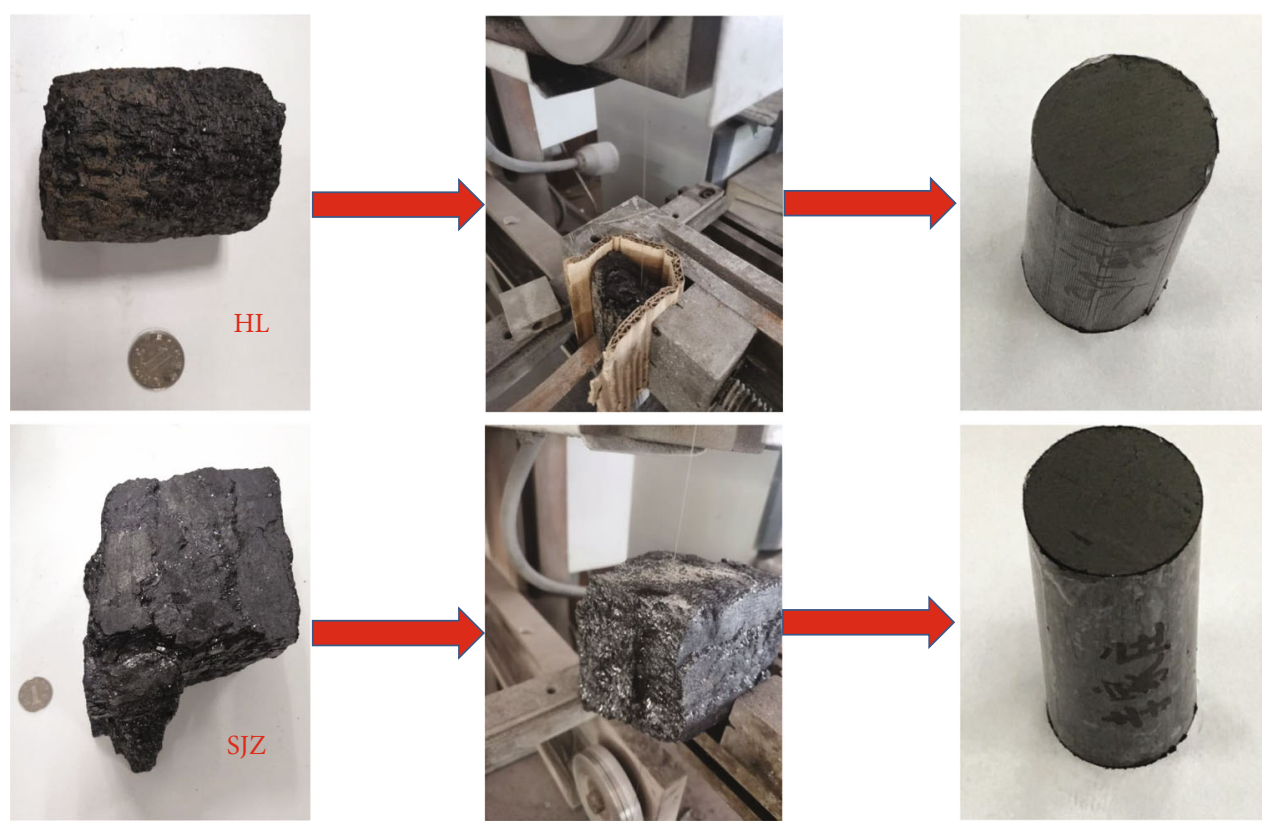

FIgURE 1: Coal sample wire-electrode cutting.

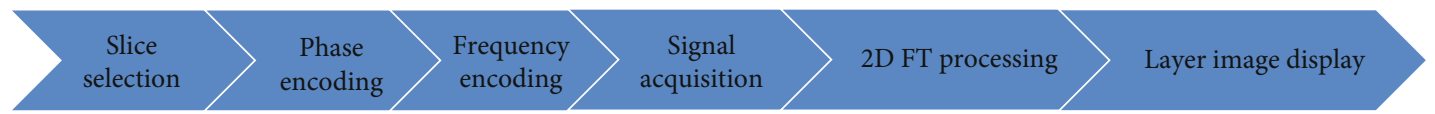

FIGURE 2: Flowchart of nuclear magnetic resonance imaging.
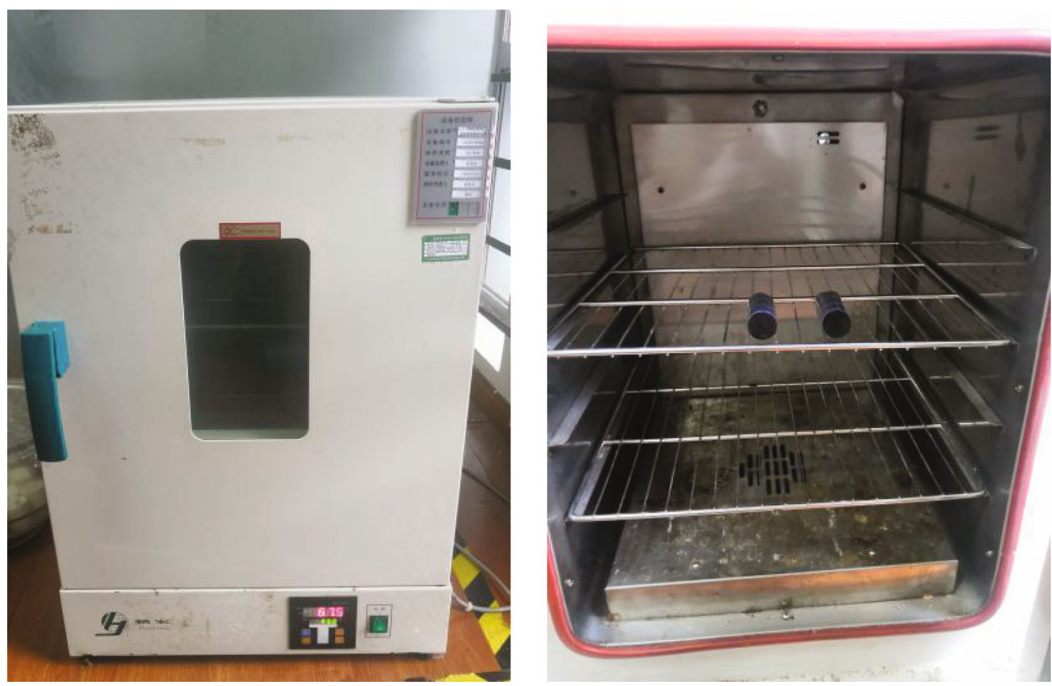

FIGURE 3: Drying oven for coal samples.

2.2.2. Experimental Procedures. The coal samples were dried at $60^{\circ} \mathrm{C}$ for 24 hours before liquid nitrogen freeze-thaw cycling. The drying oven is shown in Figure 3 . The $T_{2}$ spectrum of the dried samples was used as the base signal. The SEM, porosity, and permeability tests were carried out before the freeze-thaw cycling. Then, vacuum-water saturation treatment was performed (12 hours). The vacuumwater saturation device is shown in Figure 4 . The $T_{2}$ spectrum and NMRI tests were conducted under water satura- tion, and then, the sample was dried $\left(60^{\circ} \mathrm{C}\right.$ for 24 hours). Next, the liquid nitrogen freeze-thaw cycles and related tests were carried out, using liquid nitrogen to freeze the sample for 1 hour. Figure 5 shows the liquid nitrogen freeze-thaw device and frozen samples. Then, the samples were melted at room temperature for 1 hour, and vacuum-water saturation treatment was carried out (12 hours) after every two liquid nitrogen freeze-thaw cycles. The $T_{2}$ spectrum test was performed under water saturation, and then, the samples 

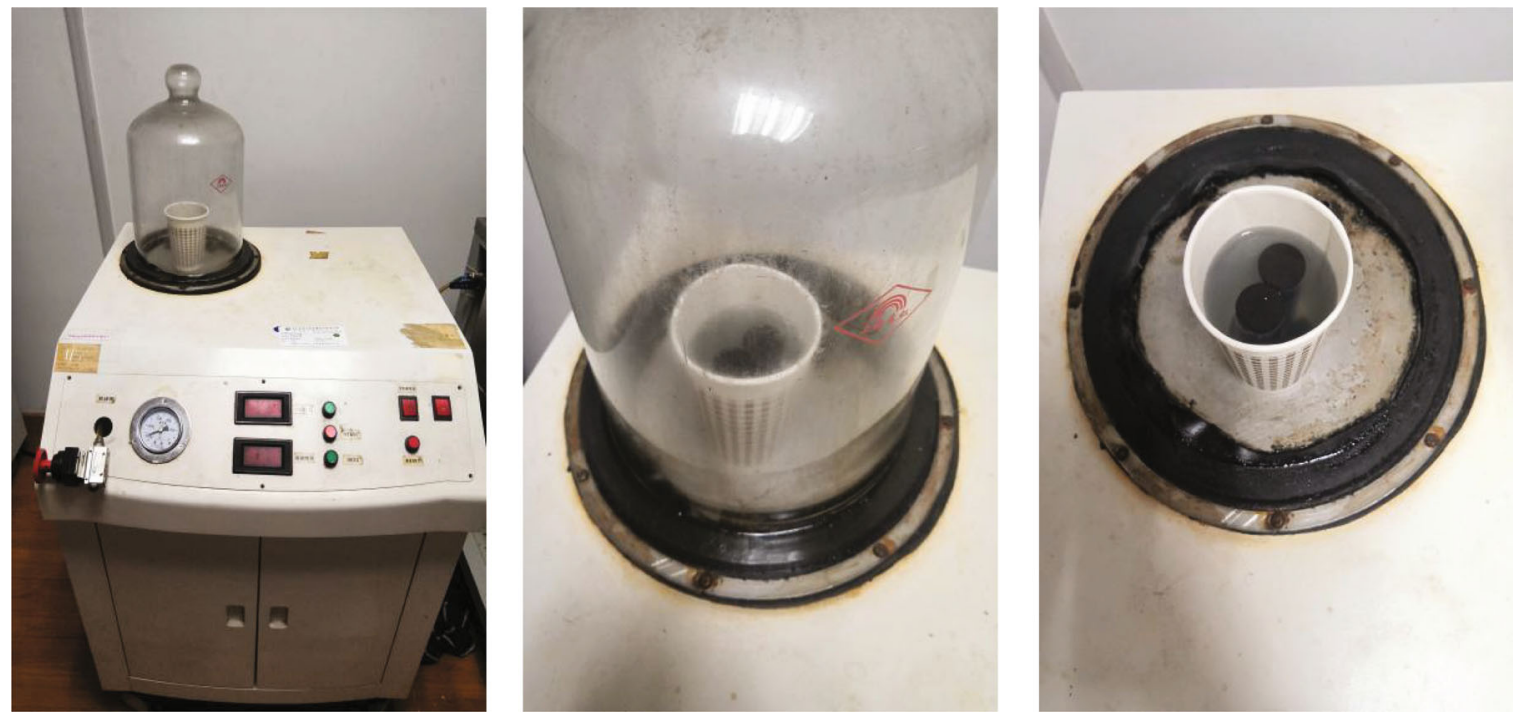

Figure 4: Vacuum-water saturation device.
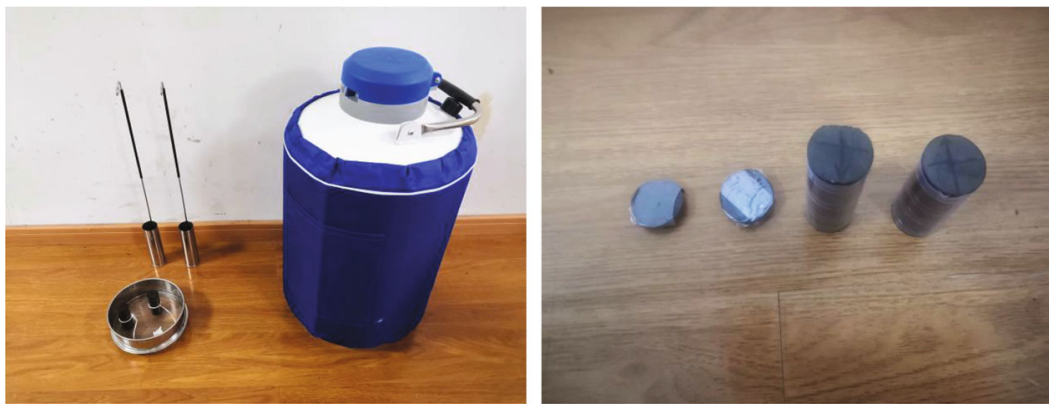

FIGURE 5: Liquid nitrogen freeze-thaw device and frozen samples.

were dried at $60^{\circ} \mathrm{C}$ for 24 hours and the porosity and permeability tests were conducted. Finally, after 10 liquid nitrogen freeze-thaw cycles, NMRI and SEM imaging of the samples were implemented.

\section{Results and Discussion}

3.1. SEM Comparison before and after Freeze-Thaw Cycles. The deep and shallow coal samples were imaged before and after the freeze-thaw cycles using an EVO scanning electron microscope customized from Zeiss, to investigate the effect of freeze-thaw cycles on coal microstructure, as shown in Figure 6.

As can be seen in Figure 6, the expansion of fractures can be observed in the deep coal sample (HL), and the formation of new fractures can be observed in the shallow sample (SJZ), after the liquid nitrogen freeze-thaw cycles. Therefore, the influence of the freeze-thaw cycles on the microstructure evolution of deep and shallow coal mainly manifests the formation of new fractures and the expansion of original fractures, which verifies the improved connectivity between mesopores and macropores and the fracture (macropores in NMR tests) development found in subsequent NMR tests after the liquid nitrogen freeze-thaw cycles. Zhai et al. observed the formation of new fractures in coal by SEM after freeze-thaw cycles [31]. Qin et al. also observed the expansion of fractures in coal by SEM after freeze-thaw cycles [28].

\subsection{NMRI Comparison before and after Liquid Nitrogen} Freeze-Thaw Cycles. In order to visually display the microstructure evolution of the deep and shallow coal before and after the freeze-thaw cycle, NMRI was carried out on the HL (deep) and SJZ (shallow) samples before and after 10 liquid nitrogen freeze-thaw cycles. The MesoMR23-60H medium-size NMRI analyzer developed by the Suzhou Niumag Analysis Instrument Corporation was used in this paper, as shown in Figure 7. In NMRI, the center frequency is $21.240 \mathrm{MHz}$, the echo time is $5.885 \mathrm{~ms}$, the repetition time is $500 \mathrm{~ms}$, the slice thickness is $25 \mathrm{~mm}$, the stacking times is 16 , the frequency code is 256, and the phase code is 192 .

The NMRI results are shown in Figure 8. The bright spots represent the development of the pore structure in the NMRI images. The brighter and larger spots reflect the more developed pore structure [38]. Comparing the NMRI images of deep and shallow samples before and after the freeze-thaw cycle, it can be found that the pore structure of the shallow sample before cycling is better developed than that of the deep samples, and the variation in pore structure of the shallow sample is also more significant than that of the deep coal after liquid nitrogen freeze-thaw cycles, which 

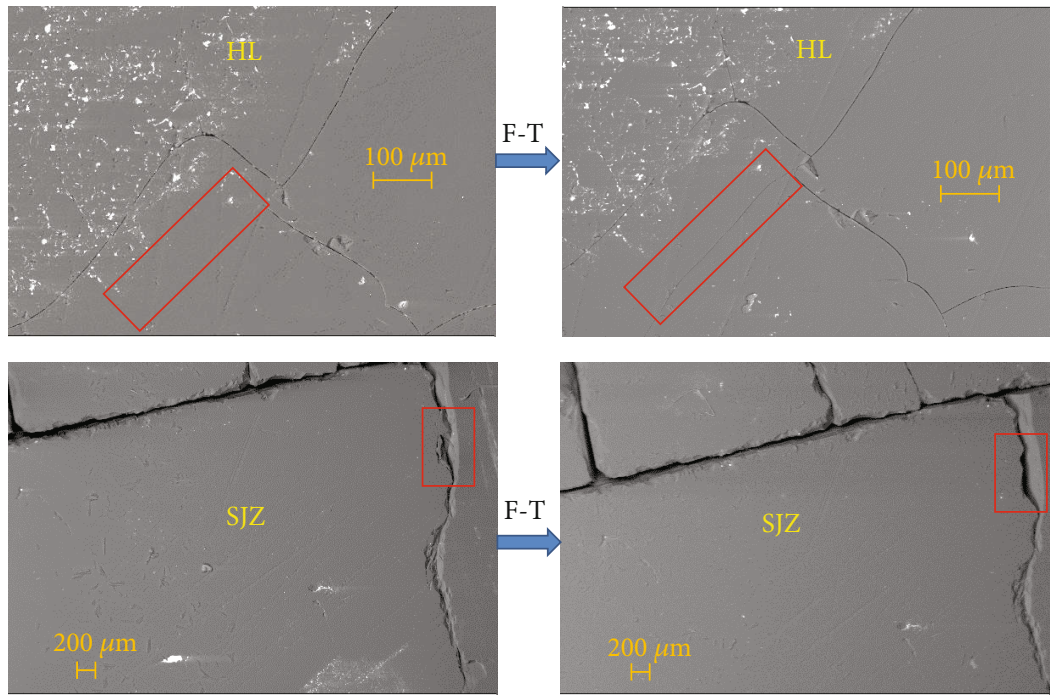

FIGURE 6: SEM images before and after freeze-thaw cycle.

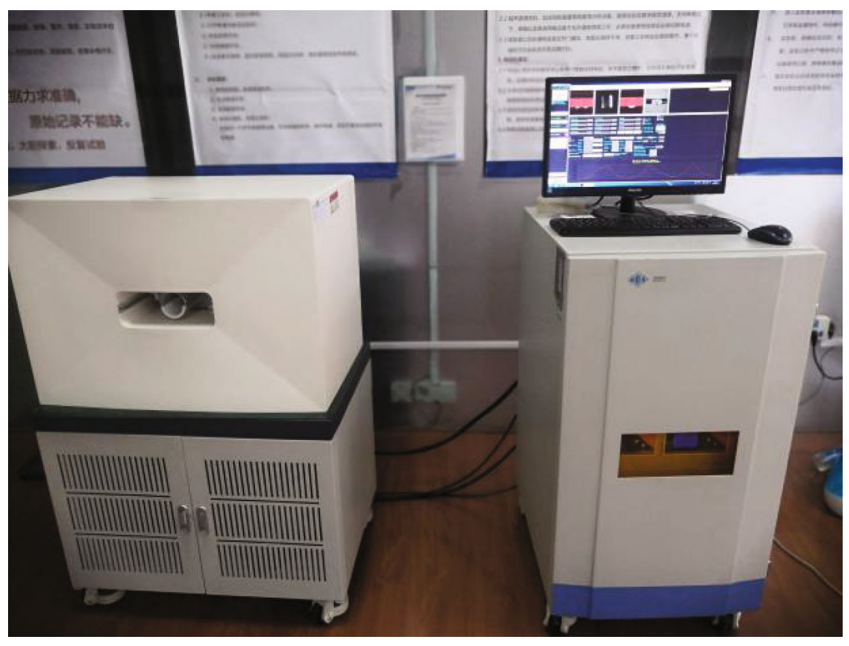

Figure 7: MesoMR23-60H medium-size NMRI analyzer.

can be seen from the initial porosity of the deep and shallow samples before the freeze-thaw cycle and the variation patterns of porosity during the freeze-thaw cycles (Figure 9).

\subsection{Evolution Law of Multiscale Coal Structure during} Liquid Nitrogen Freeze-Thaw Cycles. Considering that the NMR $T_{2}$ spectrum comprehensively can reflect the multiscale micropore, mesopore, and macropore structure characteristics, the NNM $T_{2}$ spectra of the deep (HL) and shallow (SJZ) samples were tested before freeze-thaw and after the 2nd, 4th, 6th, 8th, and 10th cycles. The MesoMR23-60H medium-size NMRI analyzer developed by Suzhou Niumag Analysis Instrument Corporation was also used in the NMR $T_{2}$ spectrum test. In the test, the main value of the $\mathrm{RF}$ signal frequency was $23 \mathrm{MHz}$, the RF signal frequency offset was $401587.9 \mathrm{~Hz}$, the RF $90^{\circ}$ pulse width was $10.00 \mu \mathrm{s}$, and the RF $180^{\circ}$ pulse width was $19.52 \mu \mathrm{s}$; the signal frequency range and the sampling frequency received by the receiver during sampling were $250 \mathrm{kHz}$, the sampling repeat interval was $3000 \mathrm{~ms}$, the RF delay was $0.08 \mathrm{~ms}$, the analog gain was 20 , the digital gain was 3 , the count of cumulative samples was 16 , the preamplifier gain was 0 , the number of echoes was 10000 , and the echo time was $0.20 \mathrm{~ms}$.

Figure 10 shows three peaks in the $T_{2}$ spectrum during liquid nitrogen freeze-thaw cycles for the HL and SJZ samples. Based on the relationship between pore size and relaxation time (Equation (1)), with the increase of relaxation time, the three peaks in the $T_{2}$ spectrum represent micropores, mesopores, and macropores, from left to right, respectively. From the evolution law of the $T_{2}$ spectrum in the process of liquid nitrogen freeze-thaw cycles, it can be found that with the increase of the number of freeze-thaw cycles, the peak areas corresponding to micropores, mesopores, and macropores increase. Namely, with the increase of the number of liquid nitrogen freeze-thaw cycles, the volumes of micropores, mesopores, and macropores increase, which can be verified by Figure 9 (variation law of porosity over freeze-thaw cycles). Also, the valleys between the peaks corresponding to mesopores and macropores gradually become less visible with more freeze-thaw cycles, which indicates that the connectivity between mesopores and macropores is gradually enhanced [38].

In order to investigate the individual evolution of micropores, mesopores, and macropores, the proportion of the peak areas corresponding to micropores, mesopores, and macropores in the total area of the three peaks after different cycle numbers is calculated over the process of liquid nitrogen freeze-thaw cycles, as shown in Figure 11.

It can be observed from Figure 11 that the proportion of micropores in the total pore volume decreases with the increase of freeze-thaw cycles, and the proportion of mesopores and macropores in the total pore volume increases. Compared to macropores, mesopores increase more obviously. Zhai et al. investigated the coal pore structure evolution under freeze-thaw cycles by NMR; they also observed that the proportion of micropores decreased and the proportion of mesopores and macropores increased [31]. 


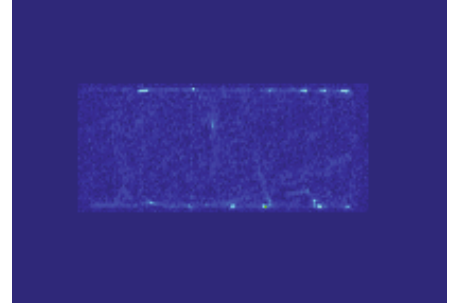

(a) NMRI of HL sample before F-T

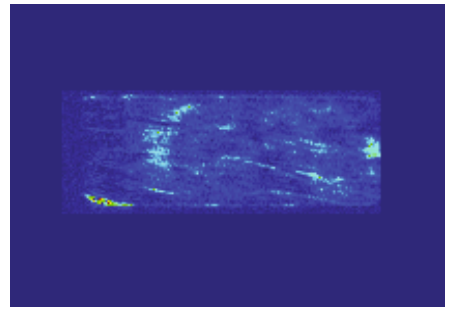

(c) NMRI of SJZ sample before F-T

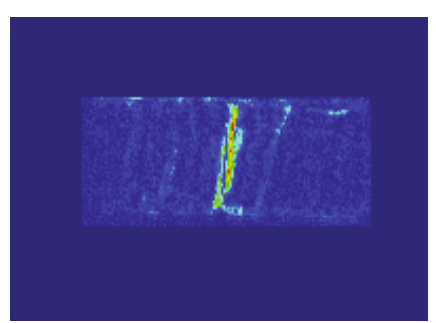

(b) NMRI of HL sample after F-T

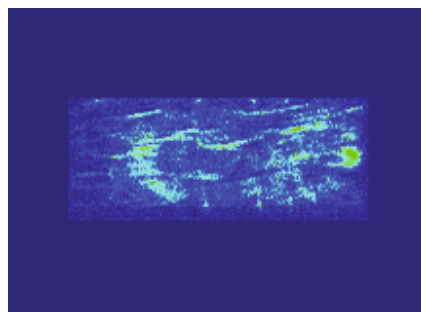

(d) NMRI of SJZ sample after F-T

FIGURE 8: Comparison of NMRI before and after freeze-thaw cycle.

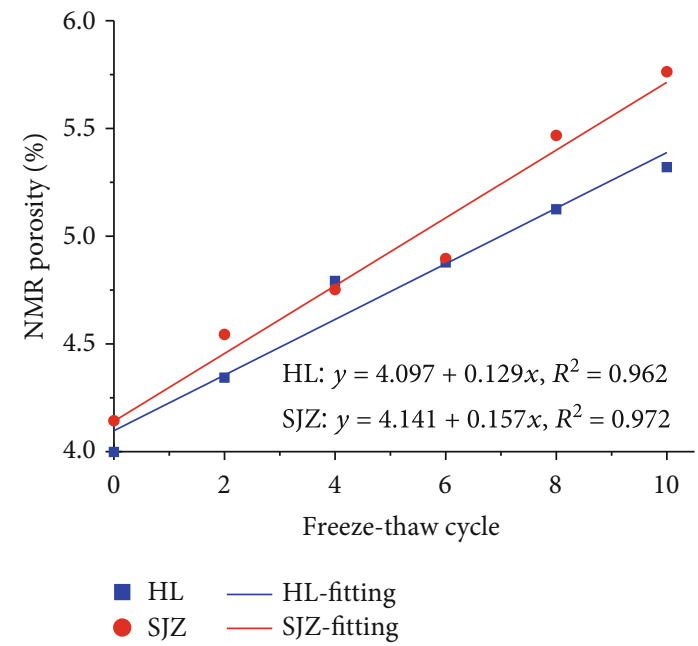

(a)

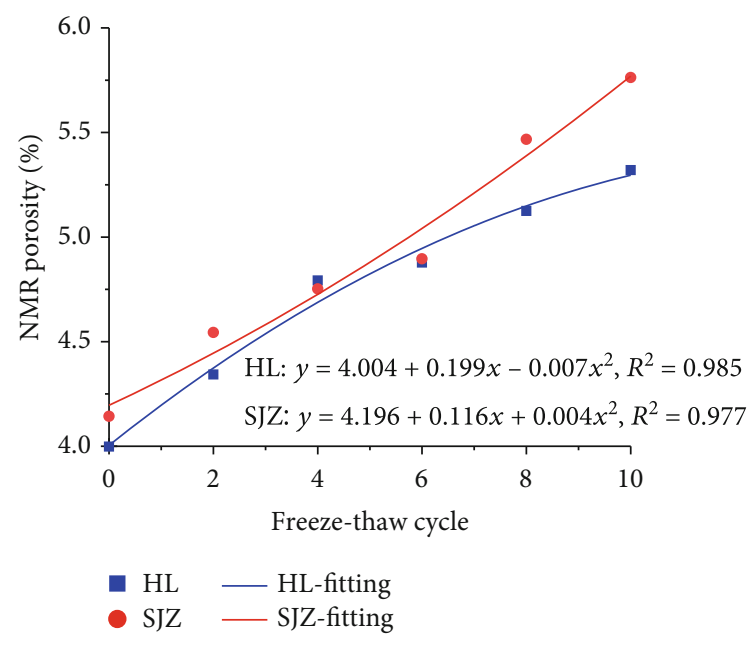

(b)

FIGURE 9: Evolution of the porosity measured by NMR during liquid nitrogen freeze-thaw cycle.

There is a linear relationship between NMR signal intensity and water mass in pore structure [39]. In view of this, this paper investigates the linear relationship between NMR signal intensity and water mass in the pore structure of the coal sample, as shown in Figure 12.

After obtaining the relationship between NMR signal intensity and water mass, the mass of water in the coal sample pore structure can be obtained by NMR signal intensity, from which the volume of the pore structure in the sample can be further obtained. Finally, the evolution law of NMR porosity with the number of freeze-thaw cycles was obtained, as shown in Figure 9.

Figure 9 indicates that the porosity of the deep (4.00\%) sample before liquid nitrogen freeze-thaw cycling is less than that of the shallow (4.14\%) sample. A linear positive correla- tion exists between the porosity of both samples and the number of freeze-thaw cycles, and the growth rate of the deep coal sample is less than that of the shallow coal sample (Figure 9(a)). Qin et al. found that the coal with larger initial porosity has a larger porosity growth rate during freeze-thaw cycling [34]. In this study, the initial porosity in shallow coal is larger than that in deep coal, which can explain the growth rate of shallow coal being larger than that of deep coal. Sun et al. and Qin et al. found that there is a quadratic function relation between the porosity and the number of freezethaw cycles in shallow coal and shallow rock, respectively $[25,26]$. Therefore, in this study, the relationship between the porosity and the number of freeze-thaw cycles was also fitted by the quadratic function (Figure 9(b)). It can be found that the quadratic function is better than the linear function 

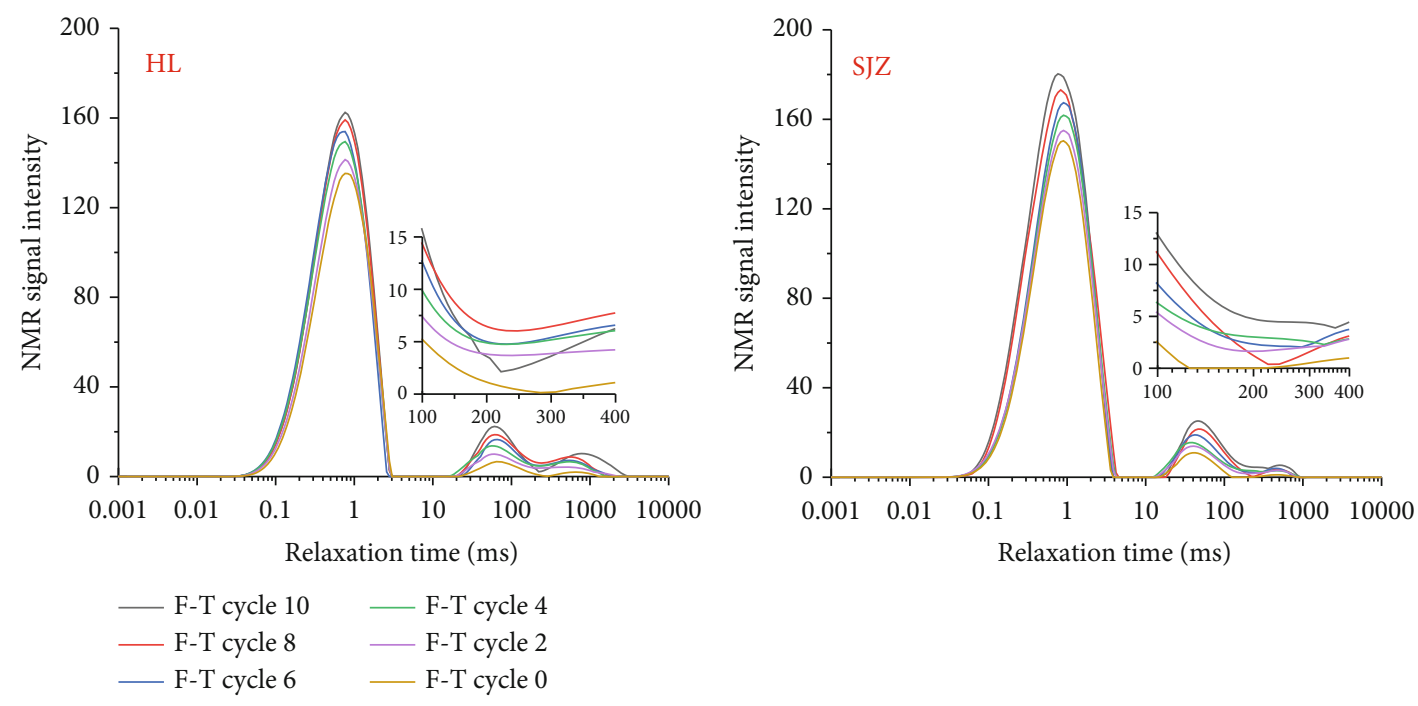

FIGURE 10: Evolution of NMR $T_{2}$ spectrum during liquid nitrogen freeze-thaw cycle.
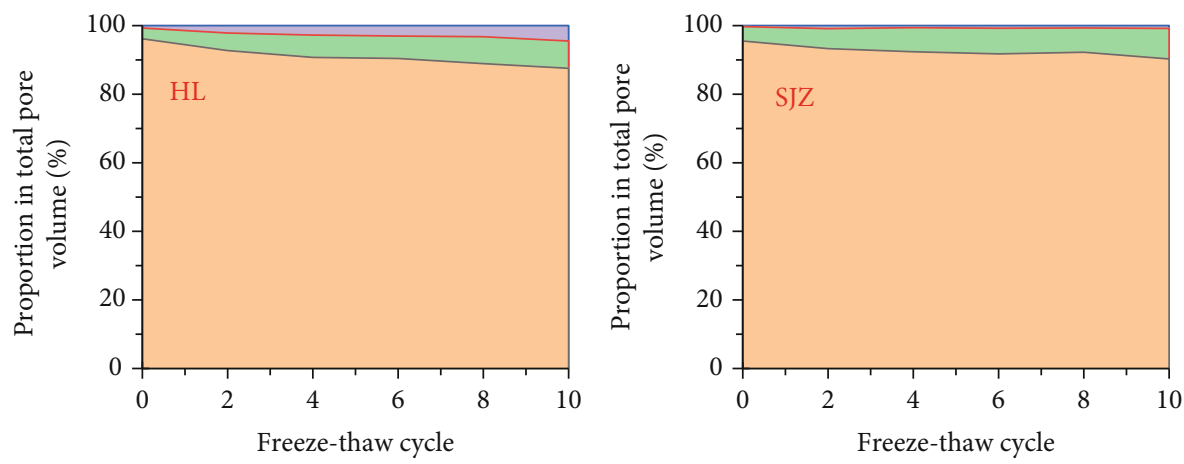

Micropores

Mesopores

Macropores

FIGURE 11: Evolution of pore structure at different scales during liquid nitrogen freeze-thaw cycles.

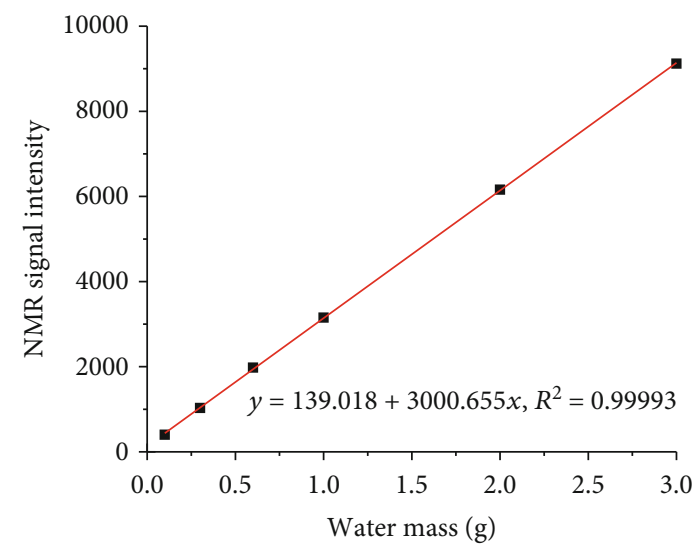

FIGURE 12: Relationship between NMR signal intensity and water mass.

in fitting the relation between the porosity and the number of freeze-thaw cycles in both deep and shallow coals. The coefficient of the quadratic term is positive in shallow coal, while the coefficient of quadratic term is negative in deep coal, which indicates that the effect of increasing the freeze-thaw number on improving the structure is more significant in shallow coal than in deep coal. The difference between deep and shallow coals in response to freeze-thaw cycles may be also caused by the difference of rock mechanical properties. $\mathrm{Li}$ and $\mathrm{Li}$ found that the compressive strength, shear strength, and cohesion of rock increase with the increase of depth [40].

\section{Conclusions}

The multiscale structure evolution law of deep and shallow coal samples from the same seam in the Qinshui coalfield during the liquid nitrogen freeze-thaw cycles was investigated using the NMR $T_{2}$ spectrum, NMRI, and SEM. The following conclusions can be drawn:

(1) The initial NMR porosity of deep coal is lower than that of shallow coal from the same coalfield and coal seam 
(2) The connectivity between mesopores and macropores in deep and shallow coal is both improved after liquid nitrogen freeze-thaw cycles, and the connectivity is gradually enhanced over freeze-thaw cycles

(3) The influence of the liquid nitrogen freeze-thaw cycle on the structure evolution of deep and shallow coal is mainly the formation and expansion of microscopic fractures

(4) During the liquid nitrogen freeze-thaw cycles, the volumes of micropores, mesopores, and macropores in deep and shallow coal increase. The proportion of micropores in the total pore structure volume decreases, and the proportion of mesopores and macropores in the total pore structure volume increases. Compared to macropores, the increase of the proportion of the mesopore volume in the total pore volume is more significant

(5) The NMR porosity of both deep and shallow coal samples increases with the number of freeze-thaw cycles, and the NMR porosity growth rate of the deep sample is lower than that of the shallow sample

\section{Data Availability}

The experimental data used to support the findings of this study are included within the article.

\section{Conflicts of Interest}

The authors declare that they have no conflicts of interest.

\section{Acknowledgments}

The research is financially sponsored by the National Natural Science Foundation of China (Grant Nos. 52004293, U1910206, 51861145403, and 51874312); the Open Project Program of Key Laboratory of Deep Earth Science and Engineering (Sichuan University), Ministry of Education (No. DESE 202004); the Fundamental Research Funds for the Central Universities (No. FRF-TP-20-034A1); the China Postdoctoral Science Foundation (No. 2018M641526); and the Yue Qi Distinguished Scholar Project of the China University of Mining \& Technology (Beijing).

\section{References}

[1] S. Li, D. Z. Tang, H. Xu, and S. Tao, "Progress in geological researches on the deep coalbed methane reservoirs," Earth Science Frontiers, vol. 23, no. 3, pp. 10-16, 2016.

[2] L. J. Zhao and Y. Qin, "Current status on deep coalbed methane in China," China Coalbed Methane, vol. 7, no. 2, pp. 3840, 2010

[3] R. R. Tonnsen and J. L. Miskimins, "Simulation of deep coalbed methane permeability and production assuming variable pore volume compressibility," Proceedings of Canadian Unconventional Resources and International Petroleum Con- ference, Society of Petroleum Engineers, Calgary, Alberta, Canada, 2010.

[4] D. D. Rice, "Composition and origins of coalbed gas," $A A P G$ Studies in Geology, vol. 38, pp. 159-184, 1993.

[5] R. C. Johnson and R. M. Flores, "Developmental geology of coalbed methane from shallow to deep in Rocky Mountain basins and in Cook Inlet-Matanuska basin, Alaska, U.S.A. and Canada," International Journal of Coal Geology, vol. 35, no. 1-4, pp. 241-282, 1998.

[6] R. R. Tonnsen and J. L. Miskimin, "A conventional look at an unconventional reservoir: coalbed methane production potential in deep environments," in Proceedings of AAPG Annual Convention and Exhibition, New Orleans, Louisiana, 2010.

[7] C. R. Nelson, "Deep coalbed gas plays in the U.S. rocky mountain region," in Proceedings of AAPG Annual Meeting, Salt Lake City, Utah, 2003.

[8] C. B. Che, Report on the New Round of National Oil and Gas Resource and Coalbed Methane Resource Evaluation Results, Beijing, 2006.

[9] C. H. Liu, J. Zhu, C. B. Che, H. L. Yang, and M. Z. Fan, "Methodologies and results of the latest assessment of coalbed methane resources in China," Natural Gas Industry, vol. 29, no. 11, pp. 130-132, 2009.

[10] Y. T. Wang and W. F. Lan, "Origin of hydrocarbons and its exploration in Cainan oilfield," Xinjiang Petroleum Geology, vol. 15, no. 1, pp. 30-36, 1994.

[11] S. H. Cui, H. L. Liu, B. Wang, N. Ning, and S. X. Sang, "Trapping characteristics of coalbed methane in low-rank coal of Zhungaer basin," Geoscience, vol. 21, no. 4, pp. 719-724, 2007.

[12] H. L. Qin, H. F. Yao, X. B. Jia, P. F. Li, and Y. J. Meng, "Pore and fissure characteristics of deep coal reservoir based on NMR," Coal Technology, vol. 38, no. 8, pp. 55-89, 2019.

[13] X. D. Gao, Study on porosity and permeability evolution mechanism and fracturing reconstruction feasibility of deep coal reservoirs in Linxing area, China university of Mining and Technology-Beijing, 2019.

[14] Q. L. Xu, W. H. Huang, S. H. Tang et al., "Pore structure and adsorption of deep medium-high rank coal reservoirs," Geoscience, vol. 30, no. 2, pp. 413-419, 2016.

[15] X. X. Lu, The reservoir characteristics of deep coal sean in Southern Qinshui basin, China university of Geosciences-Beijing, 2013.

[16] X. X. L. W. H. Huang, Y. P. Chen, S. R. Zhang, J. Wu, and Y. Y. $\mathrm{Xu}$, "Pore structure of deep coal seam in southern Qinshui basin," Journal of Northeast Petroleum University, vol. 39, no. 3, pp. 41-49, 2015.

[17] G. Q. Zheng, D. F. Yang, and X. L. Zhou, "Comparative study on reservoir properties of deep and shallow coal seams in Qinshui basin," Coal Technology, vol. 37, no. 3, pp. 108-110, 2018.

[18] H. X. Liang, P. Chen, L. H. Tong, and C. H. Lu, "Pore characteristics of deep part coal and significance in Huainan coalfield," Coal Geology of China, vol. 22, no. 6, pp. 5-8, 2010.

[19] M. Zhang, The variation law of deep coal measure gas reservoirs physical properties with the increase of temperature and pressure, China university of Mining and Technology, 2019.

[20] X. Y. Wen, X. Y. Yi, Y. Lu, B. S. Guan, L. Liang, and P. Liu, "Evalution and primary exploration of damage experiment of coal rock permeability caused by different fracture fluid," Petrochemical Industry Application, vol. 30, no. 3, pp. 12-15, 2011. 
[21] K. Zhu, "Impact of shale gas hydraulic fracturing development on environment," China Petroleum and Chemical Standard and Quality, vol. 36, no. 22, pp. 57-78, 2016.

[22] C. Z. Cai, F. Gao, G. S. Li, Z. W. Huang, and P. Hou, "Evaluation of coal damage and cracking characteristics due to liquid nitrogen cooling on the basis of the energy evolution laws," Journal of Natural Gas Science and Engineering, vol. 29, pp. 30-36, 2016.

[23] C. Zhai, L. Qin, S. M. Liu, J. Z. Xu, Z. Q. Tang, and S. L. Wu, "Pore structure in coal: pore evolution after cryogenic freezing with cyclic liquid nitrogen injection and its implication on coalbed methane extraction," Energy \& Fuels, vol. 30, no. 7, pp. 6009-6020, 2016.

[24] L. Qin, Pore evolution after fracturing with cyclic liquid nitrogen and the mechanism of permeability enhancing, China university of Mining and Technology, 2018.

[25] L. Qin, C. Zhai, S. M. Liu, J. Z. Xu, G. Q. Yu, and Y. Sun, "Changes in the petrophysical properties of coal subjected to liquid nitrogen freeze-thaw - a nuclear magnetic resonance investigation," Fuel, vol. 194, pp. 102-114, 2017.

[26] Y. Sun, C. Zhai, J. Z. Xu, Y. Z. Cong, L. Qin, and C. Zhao, "Characterisation and evolution of the full size range of pores and fractures in rocks under freeze-thaw conditions using nuclear magnetic resonance and three-dimensional X-ray microscopy," Engineering Geology, vol. 271, 2020.

[27] L. Qin, S. G. Li, Z. Cheng et al., "Changes in the pore structure of lignite after repeated cycles of liquid nitrogen freezing as determined by nitrogen adsorption and mercury intrusion," Fuel, vol. 267, pp. 117214-117214, 2020.

[28] L. Qin, C. Zhai, J. Z. Xu, S. M. Liu, C. Zhong, and G. Q. Yu, "Evolution of the pore structure in coal subjected to freeze -thaw using liquid nitrogen to enhance coalbed methane extraction," Journal of Petroleum Science and Engineering, vo, vol. 175, pp. 129-139, 2019.

[29] D. C. Yan, Experimental study on the effects of liquid nitrogen freezing and thawing damage in coal with different water content, China university of Mining and Technology, 2019.

[30] Y. Sun, C. Zhai, L. Qin, J. Z. Xu, and G. Q. Yu, "Coal pore characteristics at different freezing temperatures under conditions of freezing-thawing cycles," Environmental Earth Sciences, vol. 77, p. 525, 2018.

[31] C. Zhai, S. L. Wu, S. M. Liu, L. Qin, and J. Z. Xu, "Experimental study on coal pore structure deterioration under freeze-thaw cycles," Environmental Earth Sciences, vol. 76, no. 15, p. 507, 2017.

[32] P. C. Lauterbur, "Image formation by induced local interactions: examples employing nuclear magnetic resonance," Nature, vol. 242, no. 5394, pp. 190-191, 1973.

[33] M. R. Mitchell, R. W. Tarr, T. E. Conturo, C. L. Partain, and A. E. James, "Spin echo technique selection: basic principles for choosing MRI pulse sequence timing intervals," Radiographics, vol. 6, no. 2, pp. 245-260, 1986.

[34] Suzhou Niumag Analytical Istrument Corporation, "Nuclear magnetic resonance imaging MRI-introduction to the principle of nuclear magnetic resonance imaging," https://www .niumag.com/he-ci-gong-zhen-cheng-xiang-mri-he-ci-gongzhen-cheng-xiang.html, 2016.

[35] W. Kenyon, "Nuclear magnetic resonance as a petrophysical measurement," Nuclear Geophysics, vol. 6, no. 2, pp. 153171, 1992.
[36] S. Li, D. Tang, Z. Pan, H. Xu, and W. Huang, "Characterization of the stress sensitivity of pores for different rank coals by nuclear magnetic resonance," Fuel, vol. 111, pp. 746-754, 2013.

[37] W. Looyestijin and J. Hofman, "Wettability-index determination by nuclear magnetic resonance," SPE Reservoir Evaluation and Engineering, vol. 9, no. 2, pp. 146-153, 2006.

[38] S. Li, M. K. Luo, C. J. Fan, H. J. Bi, and Y. P. Ren, "Quantitative characterization of the effect of acidification in coals by NMR and low-temperature nitrogen adsorption," Journal of China Coal Society, vol. 42, no. 7, pp. 1748-1756, 2017.

[39] Y. X. Zhao, Y. F. Sun, S. M. Liu, K. Wang, and Y. D. Jiang, "Pore structure characterization of coal by NMR cryoporometry," Fuel, vol. 190, pp. 359-369, 2017.

[40] J. R. Li and H. B. Li, "Experimental study on mechanical properties of rocks with different depths," Chinese Journal of Rock Mechanics and Engineering, vol. 21, no. S1, pp. 948-951, 2001. 